\title{
Reducing Inappropriate Laboratory Testing in the Hospital Setting: How Low Can We Go?
}

\author{
Manpreet Basuita, MD, Corey Lyle Kamen, BSc, Cheryl Ethier, BA, and Christine Soong, MD, MSc
}

\section{ABSTRACT}

Objective: Routine laboratory testing is common among medical inpatients; however, when ordered inappropriately testing can represent low-value care. We examined the impact of an evidence-based intervention bundle on utilization.

Participants/setting: This prospective cohort study took place at a tertiary academic medical center and included 6424 patients admitted to the general internal medicine service between April 2016 and March 2018.

Intervention: An intervention bundle, whose first components were implemented in July 2016, included computer order entry restrictions on repetitive laboratory testing, education, and audit-feedback.

Measures: Data were extracted from the hospital electronic health record. The primary outcome was the number of routine blood tests (complete blood count, creatinine, and electrolytes) ordered per inpatient day.

Analysis: Descriptive statistics were calculated for demographic variables. We used statistical process control charts to compare the baseline period (April 2016-June 2017) and the intervention period (July 2017-March 2018) for the primary outcome.

Results: The mean number of combined routine laboratory tests ordered per inpatient day decreased from 1.19 (SD, 0.21 ) tests to 1.11 (SD, 0.05$)$, a relative reduction of $6.7 \%$ $(P<0.0001)$. Mean cost per case related to laboratory tests decreased from $\$ 17.24$ in the pre-intervention period to $\$ 16.17$ in the post-intervention period (relative reduction of $6.2 \%$ ). This resulted in savings of $\$ 26,851$ in the intervention year.

Conclusion: A laboratory intervention bundle was associated with small reductions in testing and costs. A routine test performed less than once per inpatient day may not be clinically appropriate or possible.

Keywords: utilization; clinical costs; quality improvement; QI intervention; internal medicine; inpatient.

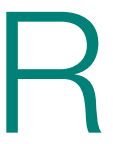
outine laboratory blood testing is a commonly used diagnostic tool that physicians rely on to provide patient care. Although routine blood testing represents less than $5 \%$ of most hospital budgets, routine use and over-reliance on testing among physicians makes it a target of cost-reduction efforts..$^{1-3} A$ variety of interventions have been proposed to reduce inappropriate laboratory tests, with varying results. ${ }^{1,4-6}$ Successful interventions include providing physicians with fee data associated with ordered laboratory tests, unbundling panels of tests, and multicomponent interventions. ${ }^{6}$ We conducted a multifaceted quality improvement study to promote and develop interventions to adopt appropriate blood test ordering practices.

\section{Methods}

\section{Setting}

This prospective cohort study took place at Mount Sinai Hospital, a 443-bed academic hospital affiliated with the University of Toronto, where more than 2400 learners rotate through annually. The study was approved by the Mount Sinai Hospital Research Ethics Board.

\section{Participants}

We included all inpatient admissions to the general internal medicine service between April 2016 and March 2018. Exclusion criteria included a length of stay (LOS) longer than 365 days and admission to a critical care unit. Patients with more than 1 admission were counted as separate hospital inpatient visits.

\section{Intervention}

Based on internal data, we targeted the top 3 most frequently ordered routine blood tests: complete blood

From the University of Toronto (Dr. Basuita, Corey L. Kamen, and Dr. Soong) and Sinai Health System (Corey L. Kamen, Cheryl Ethier, and Dr. Soong), Toronto, Ontario, Canada. Co-first authors are Manpreet Basuita, MD, and Corey L. Kamen, BSC. 


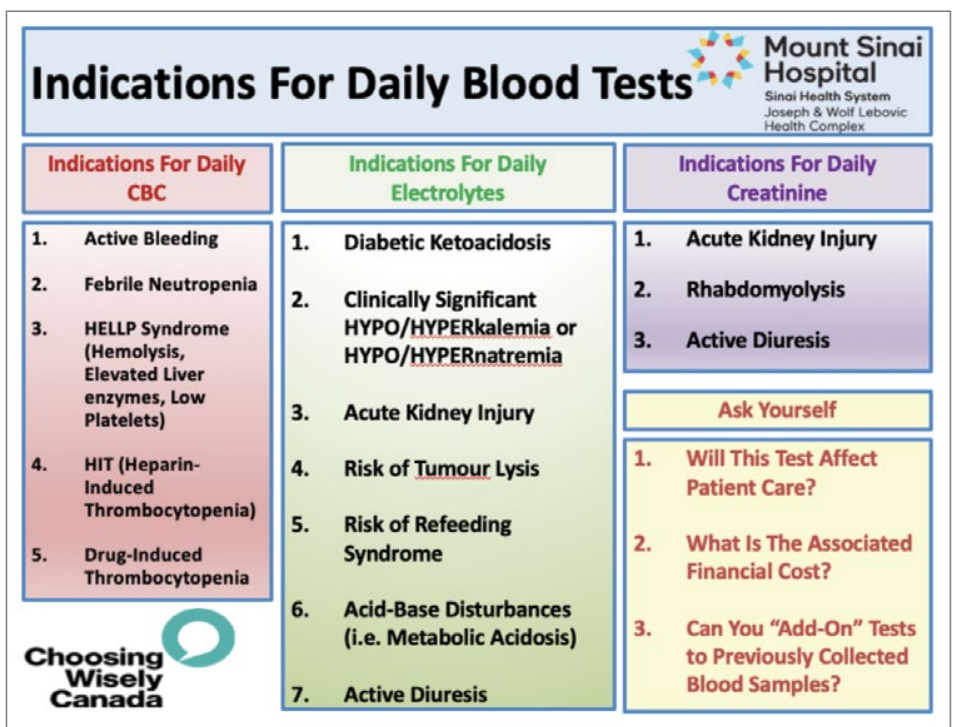

Figure 1. Educational tool displaying appropriate indications for routine daily laboratory testing based on consensus.

count (CBC), creatinine, and electrolytes. Trainee interviews revealed that habit, bundled order sets, and fear of "missing something" contributed to inappropriate routine blood test ordering. Based on these root causes, we used the Model for Improvement to iteratively develop a multimodal intervention that began in July 2016.7,8 This included a change to the computerized provider order entry (CPOE) to nudge clinicians to a restrictive ordering strategy by substituting the "Daily $\times 3$ " frequency of blood test ordering with a "Daily $x 1$ " option on a pick list of order options. Clinicians could still order daily routine blood tests for any specified duration, but would have to do so by manually changing the default setting within the CPOE.

From July 2017 to March 2018, the research team educated residents on appropriate laboratory test ordering and provided audit and feedback data to the clinicians. Diagnostic uncertainty was addressed in teaching sessions. Attending physicians were surveyed on appropriate indications for daily laboratory testing for each of $\mathrm{CBC}$, electrolytes, and creatinine. Appropriate indications (Figure 1) were displayed in visible clinical areas and incorporated into teaching sessions. ${ }^{9}$

Clinician teams received real-time performance data on their routine blood test ordering patterns compared with an institutional benchmark. Bar graphs of blood work ordering rates (sum of CBCs, creatinine, and electrolytes ordered for all patients on a given team divided by the total LOS for all patients) were distributed to each internal medicine team via email every 2 weeks (Figure 2). ${ }^{1,10-12}$

\section{Data Collection and Analysis}

Data were extracted from the hospital electronic health record (EHR). The primary outcome was the number of routine blood tests (CBC, creatinine, and electrolytes) ordered per inpatient day.

Descriptive statistics were calculated for demographic variables. We used statistical process control (SPC) charts to compare the baseline period (April 2016-June 2017) and the intervention period (July 2017-March 2018) for the primary outcome. SPC charts display process changes over time. Data are plotted in chronological order, with the central line representing the outcome mean, an upper line representing the upper control limit, and a lower line representing the lower control limit. The upper and lower limits were set at $3 \delta$, which correspond to 3 standard deviations above and below the mean. Six successive points above or beyond the mean suggests "special cause variation," indicating that observed results are unlikely due to secular trends. SPC charts are commonly used quality tools for process improvement as well as research.13-16 These charts were created using QI Macros SPC software for Excel V. 2012.07 (KnowWare International, Denver, CO).

The direct cost of each laboratory test was acquired from the hospital laboratory department. The cost of each laboratory test $(\mathrm{CBC}=\$ 7.54 /$ test, electrolytes $=\$ 2.04$ / test, creatinine $=\$ 1.28 /$ test, in Canadian dollars) was subsequently added together and multiplied by the pre- and post-intervention difference of total blood tests saved per inpatient day and then multiplied by 365 to arrive at an estimated cost savings per year.

\section{Results}

Over the study period, there were 6424 unique patient admissions on the general internal medicine service, with a median LOS of 3.5 days (Table). The majority of inpatient visits had at least 1 test of CBC (80\%; mean, 3.6 tests/visit), creatinine (79.3\%; mean, 3.5 tests/visit), 


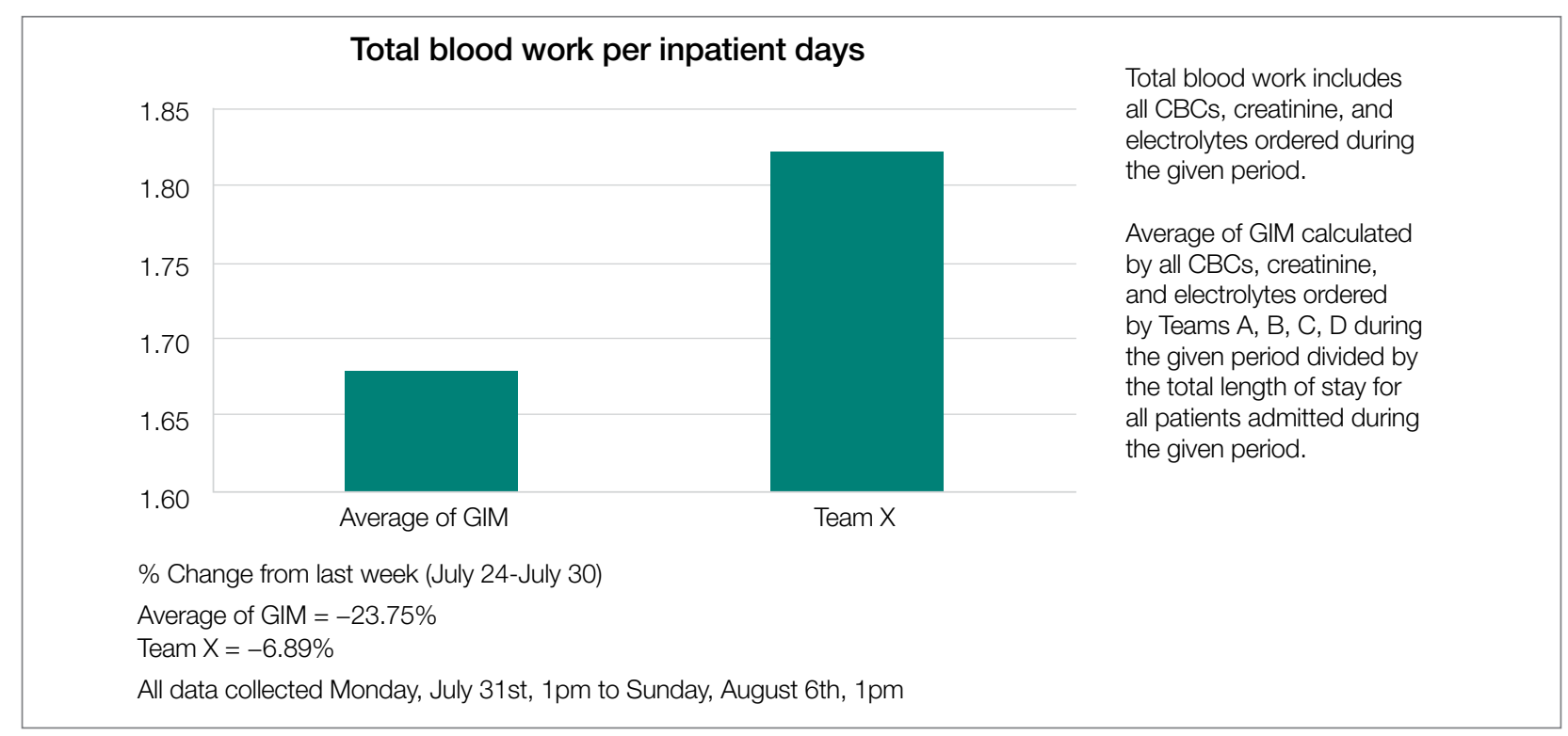

Figure 2. Sample of biweekly data distributed to each general internal medicine (GIM) team to illustrate blood work ordering patterns relative to average of all teams. $\mathrm{CBC}$, complete blood count.

or electrolytes (81.6\%; mean, 3.9 tests/visit) completed. In total, 56,767 laboratory tests were ordered.

Following the intervention, there was a reduction in both rates of routine blood test orders and their associated costs, with a shift below the mean. The mean number of tests ordered (combined CBC, creatinine, and electrolytes) per inpatient day decreased from 1.19 (SD, 0.21) in the preintervention period to 1.11 (SD, 0.05) in the post-intervention period $(P<0.0001)$, representing a $6.7 \%$ relative reduction

(Figure 3). We observed a $6.2 \%$ relative reduction in costs per inpatient day, translating to a total savings of $\$ 26,851$ over 1 year for the intervention period.

\section{Discussion}

Our study suggests that a multimodal intervention, including CPOE restrictions, resident education with posters, and audit and feedback strategies, can reduce lab test ordering on general internal medicine wards. This finding is similar to those of previous studies using a similar intervention, although different laboratory tests were targeted. 1,2,5,6,10,17

Our study found lower test result reductions than those reported by a previous study, which reported a relative reduction of $17 \%$ to $30 \%,{ }^{18}$ and by another investigation that was conducted recently in a similar setting..$^{17}$ In the latter study, reductions in laboratory testing were mostly found in nonroutine tests, and no significant improvements were noted in $\mathrm{CBC}$, electrolytes, and creatine, the 3 tests we studied over the same duration. ${ }^{17}$ This may represent a ceiling effect to reducing laboratory testing, and efforts to reduce $\mathrm{CBC}$, electrolytes, and creatinine testing beyond 0.3 to 0.4 tests per inpatient day (or combined 1.16 tests per inpatient day) may not be clinically appropriate or possible. This information can guide institutions to include other areas of overuse based on rates of utilization in order to maximize the benefits from a resource intensive intervention.

There are a number of limitations that merit discussion. First, observational studies do not demonstrate causation; however, to our knowledge, there were no other cointerventions that were being conducted during the study duration. One important note is that our project's intervention began in July, at which point there are new internal medicine residents beginning their training. As the concept of resource allocation becomes more important, medical schools are spending more time educating students about Choosing Wisely, and, therefore, newer cohorts of residents may be more cognizant of appropriate blood testing. Second, this is a single-center study, limiting generalizability; however, we note that many other centers have reported similar findings. Another limitation is that we do not know whether there were any adverse clinical events 
Table. Characteristics and Outcomes of Patients Discharged From General Internal Medicine Ward, April 2016 to March 2018

\begin{tabular}{lcc}
\hline Characteristic & Pre-intervention & Post-intervention \\
\hline No. of visits & 3162 & 3262 \\
\hline Age, mean (SD), yr & $65(21)$ & $65(21)$ \\
\hline Female, no. (\%) & $1676(53)$ & $1696(52)$ \\
\hline Length of stay, median (IQR), days & $3(0.5-5.5)$ & $4(1.5-6.5)$ \\
\hline Complete blood counts per inpatient day, mean (SD) & $0.39(0.07)$ & $0.38(0.06)$ \\
\hline Electrolytes tests per inpatient day, mean (SD) & $0.36(0.05)$ & $0.36(0.04)$ \\
\hline Creatinine tests per inpatient day, mean (SD) & $0.43(0.05)$ & $0.42(0.05)$ \\
\hline Total routine laboratory tests per inpatient day, mean (SD) & $1.19(0.21)$ & $1.11(0.05)$
\end{tabular}

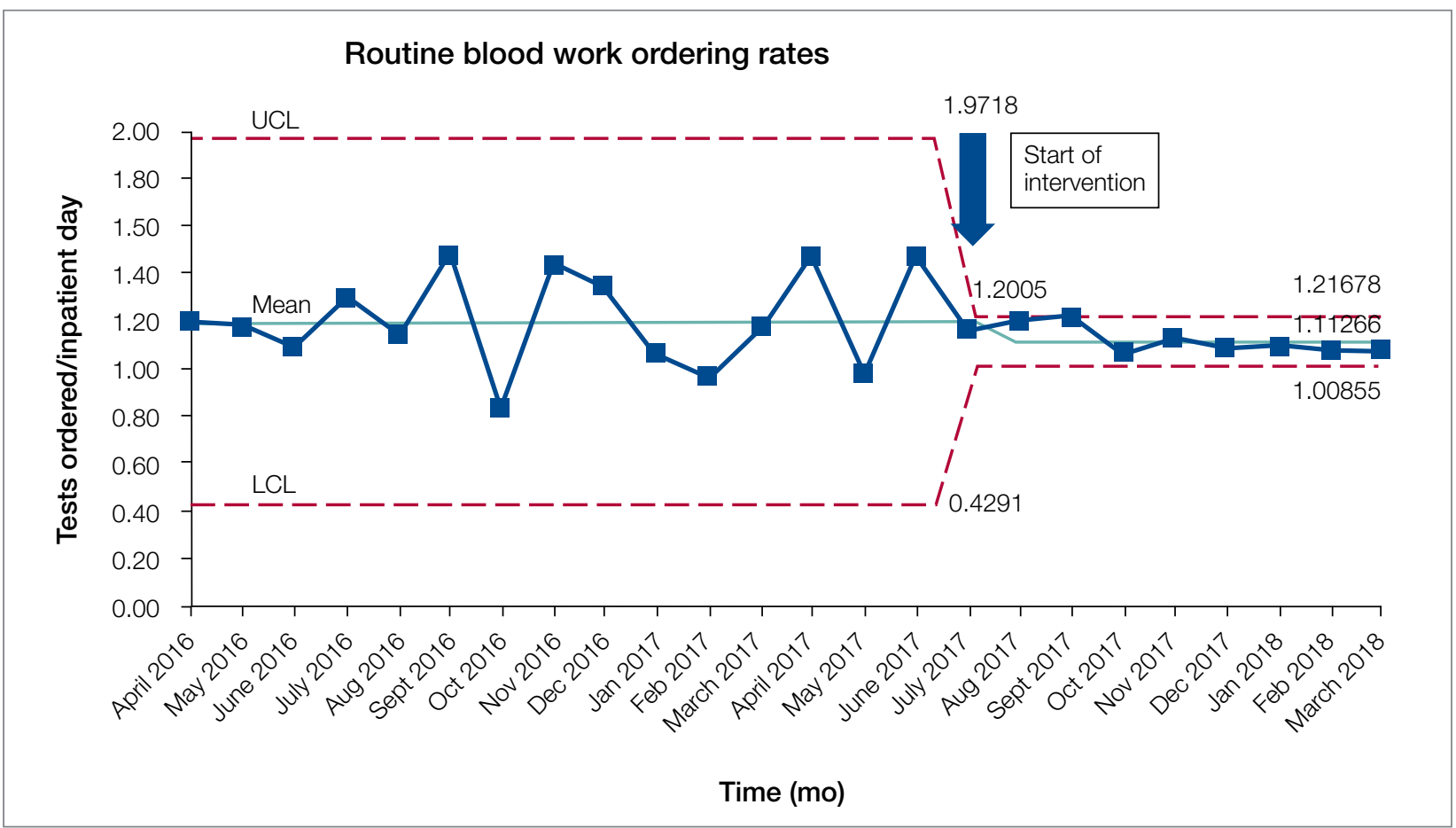

Figure 3. Routine blood work ordering rates pre- and post-intervention. LCL, lower control limit; UCL, upper control limit.

associated with blood work ordering that was too restrictive, although informal tracking of STAT laboratory testing remained stable throughout the study period. It is important to ensure that blood work is ordered in moderation and tailored to patients using one's clinical judgment.

\section{Future Directions}

We observed modest reductions in the quantity and costs associated with a quality improvement intervention aimed at reducing routine blood testing. A baseline rate of laboratory testing of less than 1 test per inpatient day may require including other target tests to drive down absolute utilization.

Corresponding author: Christine Soong, MD, MSc, 433-600 University Avenue, Toronto, Ontario, Canada M5G 1X5; Christine. soong@utoronto.ca.

Financial disclosures: None.

doi:10.12788/jcom.0027

CONTINUED ON PAGE 269 


\section{CONTINUED FROM PAGE 264}

\section{References}

1. Eaton KP, Levy $\mathrm{K}$, Soong $\mathrm{C}$, et al. Evidence-based guidelines to eliminate repetitive laboratory testing. JAMA Intern Med. 2017;178:431.

2. May TA, Clancy M, Critchfield J, et al. Reducing unnecessary inpatient laboratory testing in a teaching hospital. Am J Clin Pathol. 2006;126:200-206.

3. Thavendiranathan P, Bagai A, Ebidia A, et al. Do blood tests cause anemia in hospitalized patients? The effect of diagnostic phlebotomy on hemoglobin and hematocrit levels. J Gen Intern Med. 2005;20:520-524.

4. Feldman LS, Shihab HM, Thiemann D, et al. Impact of providing fee data on laboratory test ordering: a controlled clinical trial. JAMA Intern Med. 2013;173:903-908.

5. Attali, M, Barel $\mathrm{Y}$, Somin $\mathrm{M}$, et al. A cost-effective method for reducing the volume of laboratory tests in a university-associated teaching hospital. Mt Sinai J Med. 2006;73:787-794.

6. Faisal A, Andres K, Rind JAK, et al. Reducing the number of unnecessary routine laboratory tests through education of internal medicine residents. Postgrad Med J. 2018;94:716-719.

7. How to Improve. Institute for Healthcare Improvement. 2009. http://www.ihi.org/resources/Pages/Howtolmprove/default.aspx. Accessed June 5, 2019.

8. Langley GL, Moen R, Nolan KM, et al. The Improvement Guide: A Practical Approach to Enhancing Organizational Performance. 2nd ed. San Francisco: Jossey-Bass Publishers; 2009.

9. Hicks L. Blood Draws Toolkit. Choosing Wisely Canada. 2017. https://choosingwiselycanada.org/wpcontent/uploads/2017/10/ CWC_BloodDraws_Toolkit.pdf. Accessed March 5, 2019.

10. Sadowski BW, Lane AB, Wood SM, et al. High-value, costconscious care: iterative systems-based interventions to reduce unnecessary laboratory testing. Am J Med. 2017;130: 1112e1-1112e7.

11. Minerowicz C, Abel N, Hunter K, et al. Impact of weekly feedback on test ordering patterns. Am J Manag Care. 2015;21: 763-768.

12. Calderon-Margalit R, Mor-Yosef $\mathrm{S}$, et al. An administrative intervention to improve the utilization of laboratory tests within a university hospital. Int J Qual Health Care. 2005;17:243-248.

13. Benneyan JC, Lloyd RC, Plsek PE. Statistical process control as a tool for research and healthcare improvement. Qual Saf Health Care. 2003;12:458-64.

14. American Society for Quality. Control chart. ASM website. https:// asq.org/quality-resources/control-chart. Accessed November 5, 2020.

15. American Society for Quality. The 7 Basic Quality Tools For Process Improvement. ASM website. https://asq.org/quality-resources/ seven-basic-quality-tools. Accessed November 5, 2020.

16. Benneyan JC, Lloyd RC, Plsek PE. Statistical process control as a tool for research and healthcare improvement. Qual Saf Health Care. 2003;12:458-464.

17. Ambasta A, Ma IMY, Woo S, et al. Impact of an education and multilevel social comparison-based intervention bundle on use of routine blood tests in hospitalised patients at an academic tertiary care hospital: a controlled pre-intervention post-intervention study. BMJ Qual Saf. 2020;29:1-2.

18. Lee VS, Kawamoto K, Hess R, et al. Implementation of a value-driven outcomes program to identify high variability in clinical costs and outcomes and association with reduced cost and improved quality. JAMA. 2016;316:1061-1072. 